\title{
A Parametric Study of Viscous Fingering in Miscible Displacement by Numerical Simulation
}

by

D.E. Moissis

C.A. Miller

M.F. Wheeler

Technical Report 87-18, June 1987 



D.E. Moissis, C.A. Miller and M.F. Wheeler Rice University, Houston, $\mathrm{Tx} 77251$

Numerical simulation is used to study the effects of several parameters on miscible viscous fingering. A miscible flood of a rectangular slab. is simulated in two spatial dimensions. The parameters, obtained by the dedimensionalization of the governing equations, are the viscosity ratio, Peclet numbers associated with molecular diffusion, longitudinal dispersion and transverse dispersion and the aspect ratio of the slab. The effects of local permeability variations and the overall heterogeneity of the porous medium are also considered. A finite element modified method of characteristics is used for the solution of the concentration equation, combined with a mixed finite element method for the solution of the pressure equation. This scheme is essentially free of numerical dispersion.

The results suggest that the local permeability distribution near the entrance of the pcrous medium plays an important role in finger generation, while the permeability distribution downstream does not significantly affect fingering. The number of developing fingers and their growth rates depend strongly on the mobility ratio. The aspect ratio of the slab also influences significantly the number of fingers.

\section{Introduction}

Viscous fingering is a phenomenon that is important in miscible EOR processes. Its understanding and accurate quantitative prediction may be critical in decisions on the applicability of certain recovery processes, especially where expensive solvents are to be used.

Viscous fingers are initiated by heterogeneities in both the porous medium (permeability variations) and the fluid flow; the way they grow or are damped is a result of the combined effect of several factors, such as the mobility ratio, dispersion, gravity and the heterogeneity of the porous medium.

Several simplified models of miscible viscous fingering have been developed, in which the factors affecting viscous fingering were 
introduced as a number of parameters. Thus, koval ${ }^{2}$ proposed a one-parameter model; Todd and Longstaff proposed a 2-parameter model. More recently Fayers ${ }^{4}$ introduced a model with physically interpretable parameters. In all these models the heterogeneity of the porous medium was treated indirectly, by adjusting the value of a single parameter. However, heterogeneities can exist at different length scales, with different qualitative and quantitative effects on the displacement ${ }^{5,6}$.

In this paper we proceed with a dedimensionalization of the equations of miscible displacement to identify the dimensionless parameters that determine the solution. We then use numerical simulation to study the effect of each of these parameters on the dynamics of viscous fingering and on the displacement in general. The effects are expressed in terms of the number of fingers, their width, their locations and the root mean square growth rate.

We also study the effect of the permeability distribution and the heterogeneity of the porous medium on the generation and development of fingers. In the present paper we are interested in small scale heterogeneities, i.e. within a slab suitable for laboratory experiments.

We use a finite element modified method of characteristics ${ }^{7}$ for the approximation of the concentration of the invading fluid. The method is essentially free of numerical dispersion. It is combined with a mixed finite element method, used for the calculation of an accurate velocity field.

\section{The Model}

We assume two-dimensional, incompressible, horizontal miscible displacement in a rectangular slab of a porous medium. The pore space is initially filled with oil $(C=0)$ and flooded at the side $x=0$ with pure solvent $(\mathrm{C}=1)$. So $\mathrm{x}$ is the principal flow direction and $y$ is the direction transverse to the flow. Under these assumptions the displacement can be modeled by the following set of equations:

$$
\begin{aligned}
& u=-\frac{k}{\mu} \frac{\partial p}{\partial x}, \\
& v=-\frac{k}{\mu} \frac{\partial p}{\partial y}, \\
& \frac{\partial u}{\partial x}+\frac{\partial v}{\partial y}=0,
\end{aligned}
$$




$$
\phi \frac{\partial C}{\partial t}+u \frac{\partial C}{\partial x}+v \frac{\partial C}{\partial y}=\nabla \cdot(D \nabla C)
$$

where $D$ is the dispersion tensor $D=D\left(u, v, D, a_{\ell}, a_{t}\right), \mu=\mu\left(C, \mu_{0}, \mu_{S}\right)$ and $k=k(x, y)$. D is the molecular diffusion and $a_{l}$ and $a_{t}$ are the longitudinal and transverse mixing lengths. $\mu_{0}$ and $\mu_{s}$ are the oil and solvent viscosities respectively.

By scaling lengths in the $x$ direction by the length of the slab $L_{x}$, lengths in the $y$ direction by the width of the slab $L_{y}$, velocities by the average flowrate per unit area $q$, viscosity by the oil viscosity $\mu_{0}$, permeability by the arithmetic mean permeability $\bar{k}$, pressure by $\mathrm{g} \mu_{0} \mathrm{~L}_{\mathrm{x}} / \bar{k}$, and by expressing time in Pore Volumes Injected (PVI), the above equations are reduced to the following dimensionless form :

$$
\begin{aligned}
& u=-\frac{k}{\mu} \frac{\partial p}{\partial x}, \\
& v=-a \frac{k}{\mu} \frac{\partial p}{\partial y}, \\
& \frac{\partial u}{\partial x}+a \frac{\partial v}{\partial y}=0, \\
& \frac{\partial C}{\partial t}+u \frac{\partial C}{\partial x}+a v \frac{\partial C}{\partial y}=\nabla \cdot(D \nabla C),
\end{aligned}
$$

where: $D=D\left(u, v, P e_{m}, P e_{\ell}, P e_{t}\right), \mu=\mu(C, M)$ and $k=k(x, y)$, and the various dimensionless parameters are as defined below.

The dimensionless dispersion tensor has the following form :

$$
D=P e_{m}^{-1}\left[\begin{array}{cc}
1 & 0 \\
0 & a^{2}
\end{array}\right]+\frac{\operatorname{Pe}_{l}^{-1}}{\|u\|}\left[\begin{array}{cc}
u^{2} & a u v \\
a u v & a^{2} v^{2}
\end{array}\right]+\frac{a^{-1} \operatorname{Pe}_{t}^{-1}}{\|u\|}\left[\begin{array}{cc}
v^{2} & -a u v \\
-a u v & a^{2} u^{2}
\end{array}\right]
$$

The boundary conditions are :

$$
\begin{array}{lll}
v=0 & , \frac{\partial C}{\partial y}=0 & \text { at } y=0 \text { and } y=1 \text { (nonflow boundary), } \\
p=p_{0}(t), & , \frac{\partial C}{\partial}=1 \partial C & \text { at } x=0 \text { (inflow end) } \\
p=0 & , \frac{\partial}{\partial t}+U \frac{C}{\partial x}=0 & \text { at } x=1 \text { (outflow end). }
\end{array}
$$

The value of $p_{0}$, which is a function of time, is adjusted at each time step so that the average flowrate per unit area $q$ remains constant. 
All variables and parameters contained in the above equations are dimensionless. The parameters are the following :

Viscosity ratio: $M=\mu_{0} / \mu_{s}$, Aspect ratio of the slab: $a=L_{x} / L_{y}$

and Peclet numbers : $\mathrm{Pe}_{\mathrm{m}}=\mathrm{qL} \mathrm{L}_{\mathrm{x}} / \phi D, \mathrm{Pe}_{\ell}=\mathrm{L}_{\mathrm{x}} / \mathrm{a}_{\ell}$ and $\mathrm{Pe} \mathrm{e}_{t}=\mathrm{L}_{\mathrm{y}} / \mathrm{a}_{t} \cdot$

The three peclet numbers give the ratios of convective effects to those of molecular diffusion, longitudinal dispersion and transverse dispersion respectively.

It is clear from the above formulation that the solution depends on the values of these five parameters, as well as on the spatial dependence of permeability. In this paper numerical simulation was used to study the effect of each of these parameters on the displacement and more specifically on the number of fingers, their. locations, growth rates, and local behaviour.

As a quantitative measure of the instability of the displacement we use the root mean square (RMS) finger length for the concentration contour $C=C_{0}=0.5$, which is defined as follows:

$$
\operatorname{RMS}=\left\{\int_{0}^{1}[x(y, 0.5)-\bar{x}]^{2} d y\right\} 1 / 2
$$

where $x\left(y, C_{0}\right)$ are the $x$ coordinates of all points $(x, y)$ for which $C(x, y)=C_{0}$ and

$$
\bar{x}=\int_{0}^{1} x(y, 0.5) d y
$$

The RMS finger size is simply the standard deviation of the advance of the iso-concentration contour $\mathrm{C}=\mathrm{C}_{0}$; here $\mathrm{C}_{0}$ was chosen equal to 0.5 . This quantity is a reasonable measure of the instability of the displacement.

The porous medium considered as a base for this study was a $2 \mathrm{ft} x$ -2ft $x$ 0.5in. Berea sandstone slab, which was used in experiments by Giordano, Salter and Mohanty. They measured the permeability in each of the $1600(40 \mathrm{x} 40) 0.6 \mathrm{in}$. $\mathrm{x} 0.6 \mathrm{in}$. $\mathrm{x} 0.5 \mathrm{in}$. parallelepipeds that comprise the slab. The resulting permeability map is shown schematically in Figure l; we assumed this permeability distribution in our simulations. The permeability was assumed isotropic, with a constant value in each block. The permeability characteristics of the slab are given in Giordano et al. The arithmetic mean permeability was found to be $430 \mathrm{md}$ and the coefficient of variation (ratio of the standard deviation to the arithmetic mean) was 0.133 . The latter is a measure 
of the heterogeneity of the porous medium ${ }^{2}$ and was changed in some of our simulation runs.

The following values of the physical parameters were used in the simulation of the base case:

$\phi=0.195, M=75, D=7.5 \times 10^{-6} \mathrm{~cm}^{2} / \mathrm{sec}, a_{\ell}=2.76 \times 10^{-3} \mathrm{ft}$,

$a_{t}=7.73 \times 10^{-5} \mathrm{ft}, q=2 \mathrm{ft} /$ day.

The values of the dimensionless parameters for the various runs are given in Table 1 . The value of $\mathrm{Pe}_{\mathrm{m}}$ was the same (29409) for all runs except No.7, for which $\mathrm{Pe}_{\mathrm{m}}=5882$. In any event it was found that, for the range of the values considered, the role of molecular diffusion in determining longitudinal dispersion was negligible and in determining transverse dispersion rather small.

A quarter power mixing law was used for viscosity (dimensionless):

$$
\mu^{-1 / 4}=1+C\left(M^{1 / 4}-1\right)
$$

\section{The Numerical Method}

The coupled system of the pressure equation (Equations 1-3) and the concentration equation (4) is solved sequentially.

The solution of the concentration equation is approximated using a finite element modified method of characteristics. This scheme was first introduced by Douglas and Russell and then analysed by Russell for miscible displacement. In this algorithm the time derivative and the convective terms are combined as a directional $\tau$ derivative; time stepping is along the characteristics. The characteristics are tracked backward, using small time steps. The dispersion terms are treated implicitly using large time steps. Continuous bilinears are employed for the concentration approximation space. The truncation error is $O(\Delta t) C_{\tau \tau}+O(\Delta x)^{2}$, where $\Delta t$ is the time step and $\Delta x$ the spatial grid spacing. Since the derivative $C_{\tau \tau}$ that multiplies the $O(\Delta t)$ term is along the characteristic, it is very small and consequently it makes the first term of the above error estimate very small. The remaining term is of order $(\Delta x)^{2}$. 
This method is not conservative and in practice the time step is limited by mass balance requirements. The numerical dispersion generated by this scheme is very small; this feature is particularly important in convection-dominated problems such as the one considered here.

Because the method uses extrapolated values of velocity, high accuracy is required for the flow calculations. Therefore a mixed finite element scheme is used to solve the pressure equation (Wheeler et a.1). The method does not combine Equations (1)-(3) but solves them separately seeking a finite element approximation for both pressure and velocity. Next lowest order Raviart-Thomas spaces are used as approximating spaces. The benefit of this method is that it provides velocities, which are globally 2nd order accurate; velocities are 3 rd order accurate at certain superconvergence points. This high order of accuracy allows the use of a coarser grid than needed for standard methods. In addition, this method conserves mass in each gridblock. This method, in conjunction with the above mentioned scheme for the solution of the concentration equation, has been applied in miscible displacement problems by Ewing, Russell and Wheeler and Russell and Wheeler.

In all the simulations presented in this paper an $80 \times 80$ uniform grid was used for both pressure and concentration. Thus our simulation grid was twice as fine as the permeability grid. After some experimentation with time-stepping, a time step of 0.01 pore Volumes Injected (PVI) was selected. All simulations were run on a CRAY-XMP at Mendota Heights, MN.

\section{Effect of Permeability Distribution}

The concentration profiles (contours of constant concentration, $\mathrm{C}=0.25,0.50$ and 0.75 ) at different times are plotted in Fig. 2 for the simulation of the base case. It can be seen that at early times the profile is quite irregular with a fairly large number of small fingers (Fig. 2a). At later times (Fig. 2b) some of the initial fingers (in this case five) grow faster than the rest of the fingers and they eventually dominate the displacement. The growth of the smaller fingers is suppressed and later they merge with larger ones (Fig. $2 c, d)$, leaving unswept areas of oil that can be fairly extensive. All these processes result in a number of large fingers, which grow quite independently of each other, at least until breakthrough. These 
fingers will be referred to as "active fingers". Although the number of active fingers varies with mobility ratio, dispersion, aspect ratio and the heterogeneity of the medium, the qualitative behaviour presented here is present in all our runs.

The first question that was addressed is to what extent the permeability distribution affects the displacement at both early and later times. To relate the fingering pattern at early times to the permeability distribution near the entrance of the medium, the average (harmonic mean) permeability in the first 4 blocks in the $x$ direction was calculated for each of the 40 divisions in the $y$ direction. This permeability map was then correlated to the fingering pattern at $t=0.1$ PVI (Fig. 2a). It was observed that a finger appeared almost consistently at each block where this average permeability exhibited a (local) maximum. The largest finger appears at the block where the average permeability has its highest value. The length of the rest of the fingers however does not always correspond to the permeability value; this result is not surprising since the permeability averaging was done in a somewhat arbitrary fashion. (The idea is that the injected fluid in 0.1 PVI has covered on the average 4 permeability blocks in the $x$ direction). Furthermore this result is independent of the viscosity ratio, i.e. the number of initial fingers, their loca$t i o n s$ and their relative lengths at $t=0.1$ PVI are the same for all viscosity ratios studied, though the lengths of the fingers are, of course, different. This result strongly suggests that the permeability distribution near the entrance of the medium determines the fingering pattern at early times. This conclusion was corroborated by simulations where the slab was flooded from two of its other three sides. In all cases poor correlation was obtained when the overall average (harmonic mean) permeability was considered, i.e. when all 40 blocks in the $x$ direction were averaged.

To study the importance of the permeability distribution in the rest of the medium, a simulation was run for a slab that was homogeneous, except for the first $8 \times 40$ blocks near the entrance, i.e. the permeabilities of the remaining $32 \times 40$ blocks were replaced by a constant value, namely their arithmetic average (Run No.2). The concentration profile at $t=0.3$ PVI is shown in Fig. 3 . By comparing this to Fig. 2c, it follows that modest heterogeneity downstream has little effect on viscous fingering, other than to cause local (small wavelength) irregularities in the shape of the fingers. The effect on the number of active fingers, their locations, their lengths and the RMS 
finger growth rate is negligible. The same qualitative results were obtained in simulations with $M=7.5$. (The concentration profiles obtained in Runs No. 3 and No. 6 were approximately the same). Of course the model of the porous medium used is quite homogeneous, in the sense that the permeability variations that exist are rather small. We expect large permeability variation downstream to have a significant effect on viscous fingering.

In summary, viscous fingering is very sensitive to the permeability distribution near the entrance of the porous medium but rather insensitive to permeability variations downstream. Consequently, if viscous fingering is to be modeled correctly, two different time periods have to be considered: an initial period when permeability distribution is important and when there exists a large number of fingers and the later stage of the displacement, when permeability plays little or no role and there exists a number of active fingers, fairly independent of each other. In the former period the displacement can be modeled as a fingered mixing zone; in the latter period it can be modeled as a set of large individual fingers growing with little or no interaction.

It also follows that in unstable EOR processes the values of permeability must be known with a good degree of accuracy near the entrance of the medium (e.g. near wells and especially injection wells), if the process is to be simulated accurately; such a high degree of accuracy is not as important for the permeability of the rest of the medium.

\section{Effect of Heterogeneity of the Porous Medium}

In Run No. 4 the permeabilities of all 1600 blocks were altered according to the formula :

$$
\left.k_{\text {new }}=k_{\text {original }}-0.5 \text { (koriginal }-\bar{k}\right)
$$

This modification made the medium more homogeneous, by reducing the standard deviation $\sigma$ of the permeability distribution by half, while the value $\vec{k}$ of the average (arithmetic mean) permeability remained the same $(\bar{x}=1.00)$ and the permeability map was not qualitatively altered. Similarly, in Run No. 5 the permeabilities were altered as follows:

$$
\left.k_{n e w}=k_{\text {original }}+0.5 \text { ( } k_{\text {original }}-\bar{k}\right)
$$


The medium was now made more heterogeneous, by increasing the standard deviation by $50 \%$, while preserving the value of $\bar{k}$ and the permeability map.

The concentration profiles for $t=0.4$ PVI are shown in Figs. 4 and 5 for runs No. 4 and No. 5 respectively. By comparing Figs. 4,5 and $2 d$ it follows that heterogeneity of the medium affects the number of active fingers, the length of the fingers and the local behavior, i.e. the smoothness or irregularity of the fingers. Thus a high heterogeneity enhances merging of fingers resulting in a smaller number of active fingers ( 2 active fingers in Fig. 5 as opposed to 5 fingers in Figs. 4 and $2 d$ ). It also yields more irregularly shaped fingers. The difference between Figs. 4 and $2 d$ is rather small, except for some more splitting and merging that occurs for higher SD; however between these two and Fig. 5 there is a significant difference.

Finally the RMS finger growth rate increases with heterogeneity as shown in Fig. 6. It can be observed here that the largest difference in the growth rates for the three cases occurs in the initial 0.1 PVI. This result suggests that it is the permeability distribution near the entrance that mainly causes the difference in growth rates and demonstrates once more the importance of the permeability variations in this region.

\section{Effect of Viscosity Ratio}

The concentration profiles at $t=0.3$ PVI for Runs No. 6 and No. 7 (Viscosity ratios 7.5 and 750 respectively) are shown in Figs. 7 and 8. Comparing these to Fig. $2 \mathrm{C}(M=75)$ it follows that the behaviour of the displacement is quite different at low unfavourable viscosity ratios (Fig. 7) and at high and very high viscosity ratios (Figs. 2c and 8).

The behaviour at $M=75$ was discussed in a previous section. The main characteristic is that the large initial number of fingers is reduced by merging to a smaller number of "active fingers". The effect of the viscosity ratio on this process is very important : At low viscosity ratios (7.5) there are no fingers that grow dramatically faster than the rest; consequently merging and growth suppression occur to a much lesser extent, resulting in a larger number of active fingers, whose growth rates do not vary much. So at low viscosity ratios the 
displacement can be modeled as a fingered mixing zone even at later times. On the other hand at very high viscosity ratios (750) the active fingers start to outgrow the others earlier than for $M=75$; more merging and suppression of growth occur and this results in a smaller number of long active fingers. It can also be noticed that as the viscosity ratio increases the fingers are more unstable. Thus, for $M=7.5$ the fingers are quite smooth, for $M=75$ they are irregularly shaped and exhibit some splitting and for $M=750$ they are very irregular and exhibit large scale splitting, growing secondary fingers.

There are two causes which account for this increasing irregularity of the fingers with increasing viscosity ratio. First, the few fingers present at high viscosity ratios grow wider (in the y-direction). Hence they are more subject to small wavelength instabilities than the relatively narrow fingers that appear at low viscosity ratios. Second, the thickness of the displacement front (represented in the plots as the distance between different concentration contours) decreases as viscosity ratio increases. The relatively diffuse front which appears at low viscosity ratios is less susceptible to instabilities than the sharp front that exists at high viscosity ratios.

Fig. 9 shows how the viscosity ratio affects the RMS finger growth rate. The effect seems to be more pronounced at low values of $M$ than at high ones. As viscosity ratio tends to infinity, the RMS growth rate seems to approach an asymptotic value; this could however be a numerical effect, because a finer grid is needed for the accurate simulation of displacements with very high viscosity ratio. It can also be noticed from Fig. 9 that the RMS finger length grows almost linearly with time, except in the initial stage of the displacement. It was also found that growth of active individual fingers is also fairly linear in most cases, except again at early times. This fact is yet another indication of the different phenomena that dominate the displacement at early and at later times.

Effect of Longitudinal Dispersion

Fig. 10 shows the concentration profiles at $t=0.3$ for Run No. 8 . This, compared to Fig. 2c, illustrates the effect of longitudinal dispersion (Although, both longitudinal and transverse dispersion in Run No. 8 are 5 times larger than in the base case, the transverse dispersion is still relatively small and increasing the longitudinal 
dispersion is the dominant effect).

As expected, large longitudinal dispersion yields a thick (more diffuse) displacement front, which makes the fingers less prone to flow disturbances arising from small scale heterogeneities. In Run No. 8 longitudinal dispersion is large enough to make the fingers virtually free of small wavelength disturbances. In addition, since the front is more diffuse, the waves corresponding to small values of concentration grow faster and breakthrough occurs earlier. This may give the impression that fingers grow faster when longitudinal dispersion is larger; in fact, it is hard to define finger lengths or growth rates in this case, because the displacement front is very diffuse; what we can do is to measure RMS growth rates for different concentration contours. In our case we find that the RMS growth rate for $C_{0}=0.5$ is virtually unaffected by longitudinal dispersion. It is clear that the RMS growth rate increases with longitudinal dispersion for $\mathrm{C}_{0}<0.5$ and decreases for $\mathrm{C}_{0}>0.5$.

\section{Effect of Transverse Dispersion}

Figs. 11 and 12 show the concentration profiles at $t=0.3$ for Runs Nos. 9 and 10 to illustrate the effect of transverse dispersion at different viscosity ratios (Compare to Figs. 7 and $2 c$ respectively).

It should be noted that, although in these two runs transverse dispersion was increased by a factor of 10, its effect is still not very significant. In this range larger transverse dispersion for both low and high viscosity ratios results in shorter and wider fingers and in a thicker displacement front. The fingers are also smoother (the effect of the diffuse front more than compensates for the effect of wider fingers). The number of active fingers has not been affected by transverse dispersion in the cases studied. However merging of small fingers with bigger ones occurs faster when transverse dispersion is larger. We expect even larger transverse dispersion to cause significantly more merging, resulting in a smaller number of active fingers.

RMS finger growth rate decreases as transverse dispersion increases, as is shown in Fig. 13. The effect seems to be more pronounced at low values of $M$. Also the suppression of finger growth becomes increasingly important as transverse dispersion increases. We expect that sufficient transverse dispersion will completely wipe out 
fingering at very low viscosity ratios.

\section{Effect of Aspect Ratio}

In Run No. Il the aspect ratio of the slab was changed to 2 . The permeability distribution remained the same but the $40 \mathrm{x} 40$ permeability grid blocks were changed to rectangles of aspect ratio 2 . Likewise in Run No. 12 the aspect ratio was changed to 4 . The concentration profiles at $t=0.3$ for these two Runs are shown in Figs. 14 and 15 respectively. The effect of the aspect ratio is made quite clear by comparing these figures to Fig. $2 c$ (aspect ratio 1 ). When the slab is made narrower, the initial fingers are closer to one another and consequently their interaction is stronger. The result is that growth suppression of smaller fingers and merging occur in the initial stages of the displacement, yielding, even at early times, a small number of active fingers. In our case, for a viscosity ratio of 75 , we get five active fingers for $a=1$, two active fingers for $a=2$ and one active $f$ inger for $a=4$. The results suggest that, when the aspect ratio is large, the displacement has to be modeled as a number of individual fingers even at early times. This fact causes the RMS growth rate to increase significantly with aspect ratio, as shown in Fig. 16.

\section{Conclusions}

The factors that affect viscous fingering can be expressed as dimensionless parameters, most of which arise during the dedimensionalization of the miscible displacement equations. The main parameters are the viscosity ratio, longitudinal and transverse dispersion, the aspect ratio and the permeability characteristics of the porous medium. The effect of each parameter can be summarized as follows:

The permeability distribution near the entrance of the porous medium determines the initial number, locations and relative growth rates of the fingers; none of these depends on the viscosity ratio. Subsequent merging reduces the initial number of fingers to a smaller number of "active fingers", which continue growing through the porous medium. The permeability distribution downstream (away from the entrance) has little effect on the merging process, on the number of active fingers and on the displacement in general, provided permeability variations are not 
very large.

The root mean square finger growth rate increases with the heterogeneity of the porous medium. Also the number of active fingers decreases with increasing heterogeneity, because larger heterogeneity facilitates merging. The effect of heterogeneity is more prominent in the initial stages of the displacement.

The RMS finger growth rate increases with viscosity ratio; the effect is more prominent at low viscosity ratios than at high viscosity ratios. At low viscosity ratios little merging occurs and this results in a large number of thin active fingers with comparable growth rates. At high viscosity ratios a smaller number of wider active fingers forms. The thickness of the displacement front decreases with increasing viscosity ratio. The higher the viscosity ratio the more irregular and unstable the fingers that form.

Longitudinal dispersion causes the displacement front to be thicker and the fingers smoother. It does not affect the RMS growth rate (for the $\mathrm{C}=0.5$ concentration contour) nor the number of active fingers.

Transverse dispersion causes earlier merging. The resulting fingers are wider and somewhat smoother. RMS finger growth rate decreases with increasing transverse dispersion. The effect is stronger at low viscosity ratios.

A large aspect ratio (narrower slab) yields a smaller number of active fingers. Merging occurs early in the displacement. At very large aspect ratios a single active finger forms. RMS finger growth rate increases with aspect ratio.

\section{$\underline{\text { References }}$}

1. R.M. Giordano, S.J. Salter and K.K. Mohanty, The Effects of Permeability Variations on Flow in Porous Media, paper SPE 14365, presented at the 60th Annual Technical Conference and Exhibition of $\mathrm{SPE}$, Las Vegas, NV, September 1985.

2. E.J. Koval, A Method for Predicting the Performance of Unstable 
Miscible Displacement in Heterogeneous Media, Trans., AIME, 228 , pp. 145-154 (1963).

3. M.R.Todd and W.J.Longstaff, The Development, Testing and Application of a Numerical Simulator for Predicting Miscible Flood Performance, Trans., AIME, 253, pp. 874-882 (1975).

4. F.J. Fayers, An Approximate Model with Physically Interpretable Parameters for Representing Miscible Viscous Fingering, paper SPE 13166 presented at the 59th Annual Technical Conference and Exhibition of SPE, Houston, TX, September 1984.

5. K.J. Weber, How Heterogeneity affects Oil Recovery, Reservoir Characterization, edited by L.W. Lake and H.B. Carroll Jr., Academic, Orlando, FL, 1986, pp. 487-544.

6. T.J. Lasseter, J.R. Waggoner and L.W. Lake, Reservoir Heterogeneities and their Influence on Ultimate Recovery, Reservoir Characterization, edited by L.W. Lake and H.B. Carroll Jr., Academic, Orlando, FL, 1986, pp. 545-559.

7. J. Douglas, Jr. and T.F. Russell, Numerical Methods for Convectiondominated diffusion Problems Based on Combining the Method of Characteristics with Finite Difference or Finite Element Procedures, SIAM J. Numer. Anal., Vol. 19, No. 5, October 1982, pp. 871-884.

8. T.F. Russell, Finite Elements with Characteristics for Two-Component Incompressible Miscible Displacement, paper SPE 10500, Proc. Sixth SPE Symp. on Reservoir Simulation, New Orleans, LA, Jan-Feb 1982 .

9. M.F. Wheeler and R. Gonzalez, Mixed Finite Element Methods for Petroleum Reservoir Engineering Problems, Computing Methods in Applied Sciences and Engineering VI, edited by R. Glowinski and J.L. Lions, North Holland, New York, 1984, pp. 639-658.

10. M. Nakata, A. Weiser and M.F. Wheeler, Some Superconvergence Results for Mixed Finite Element Methods for Elliptic Problems on Rectangular Domains, The Mathematics of Finite Elements and Applications V, MAFELAP 1984, edited by J.R. Whiteman, Academic, London, 1985, pp. 367-389.

11. R.E. Ewing, T.F. Russell and M.F. Wheeler, Simulation of Miscible Displacement Using Mixed Methods and a Modified Method of Characteristics, paper SPE 12241, presented at the Reservoir Simulation Symposium, San Francisco, CA, November 1983.

12. T.F. Russell and M.F. Wheeler, Finite Element and Finite Difference Methods for Continuous Flows in Porous Media The Mathematics of Reservoir Simulation, edited by R.E. Ewing, SIAM, Philadelphia, PA, 1983, pp.35-105. 
13. T.F. Russell, M.F. Wheeler and C. Chiang, Large-Scale Simulation of Miscible Displacement by Mixed Characteristic Finite Element Methods, Mathematical Computational Methods in Seismic Exploration and Reservoir Modeling, edited by W.E. Fitzgibbons, SIAM, Philadelphia, 1986, pp. 85-107. 
Table 1. Simulation Runs

\begin{tabular}{|c|c|c|c|c|c|}
\hline Run & $M$ & $a$ & $\mathrm{Pe}_{1}$ & $P e_{t}$ & $\sigma$ \\
\hline 1 & 75 & 1 & 725 & 25873 & 0.133 \\
\hline 2 & 75 & 1 & 725 & 25873 & $0.063^{*}$ \\
\hline 3 & 7.5 & 1 & 725 & 25873 & $0.063^{*}$ \\
\hline 4 & 75 & 1 & 725 & 25873 & 0.067 \\
\hline 5 & 75 & 1 & 725 & 25873 & 0.201 \\
\hline 6 & 7.5 & 1 & 725 & 25873 & 0.133 \\
\hline 7 & 750 & 1 & 725 & 25873 & 0.133 \\
\hline 8 & 75 & 1 & 145 & 5175 & 0.133 \\
\hline 9 & 7.5 & 1 & 725 & 2587 & 0.133 \\
\hline 10 & 75 & 1 & 725 & 2587 & 0.133 \\
\hline 11 & 75 & 2 & 725 & 25873 & 0.133 \\
\hline 12 & 75 & 4 & 725 & 25873 & 0.133 \\
\hline
\end{tabular}

(*) Uniform permeability in $32 \times 40$ downstream blocks

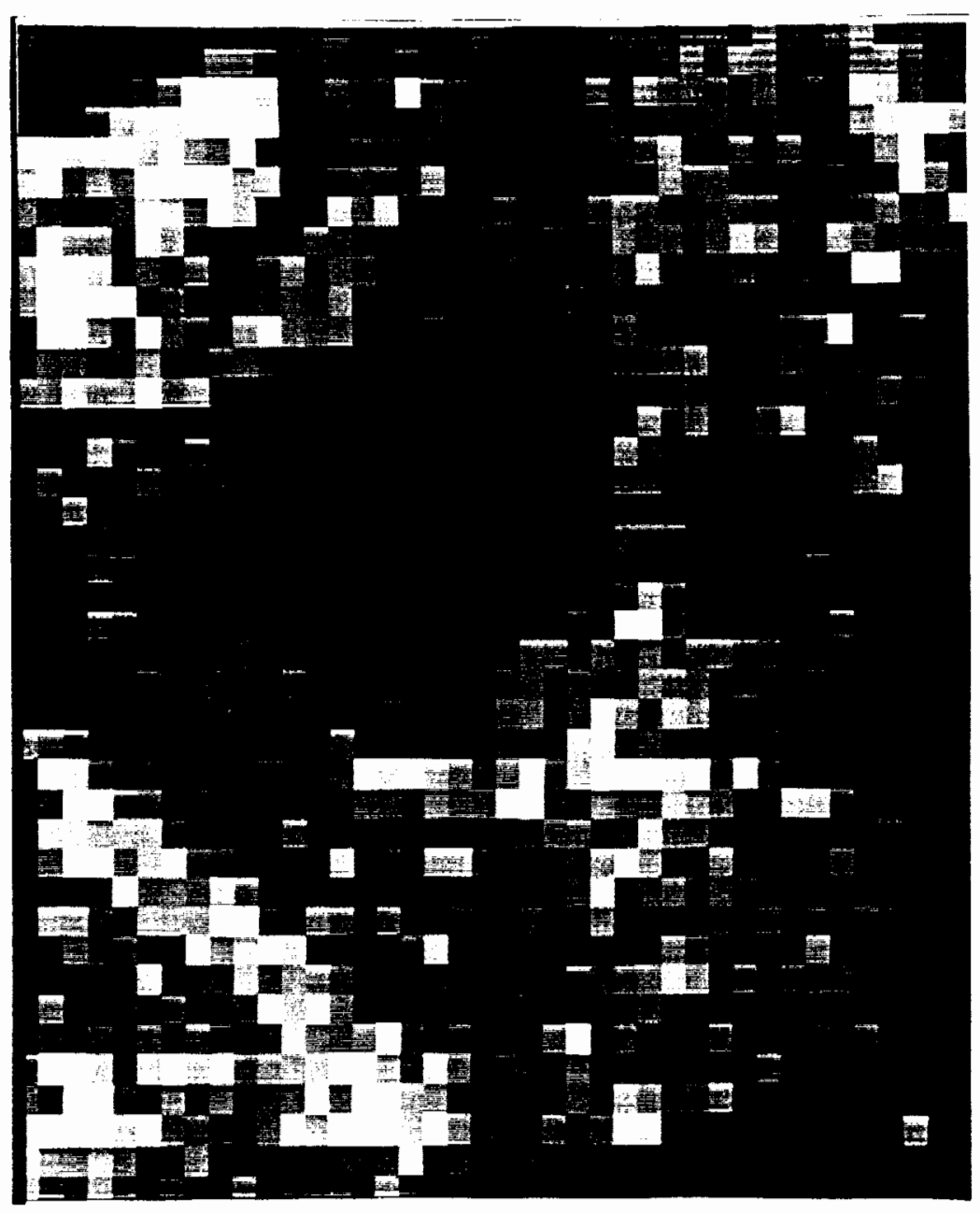

Fig. 1. Permeability map of the slab used in the simulations 


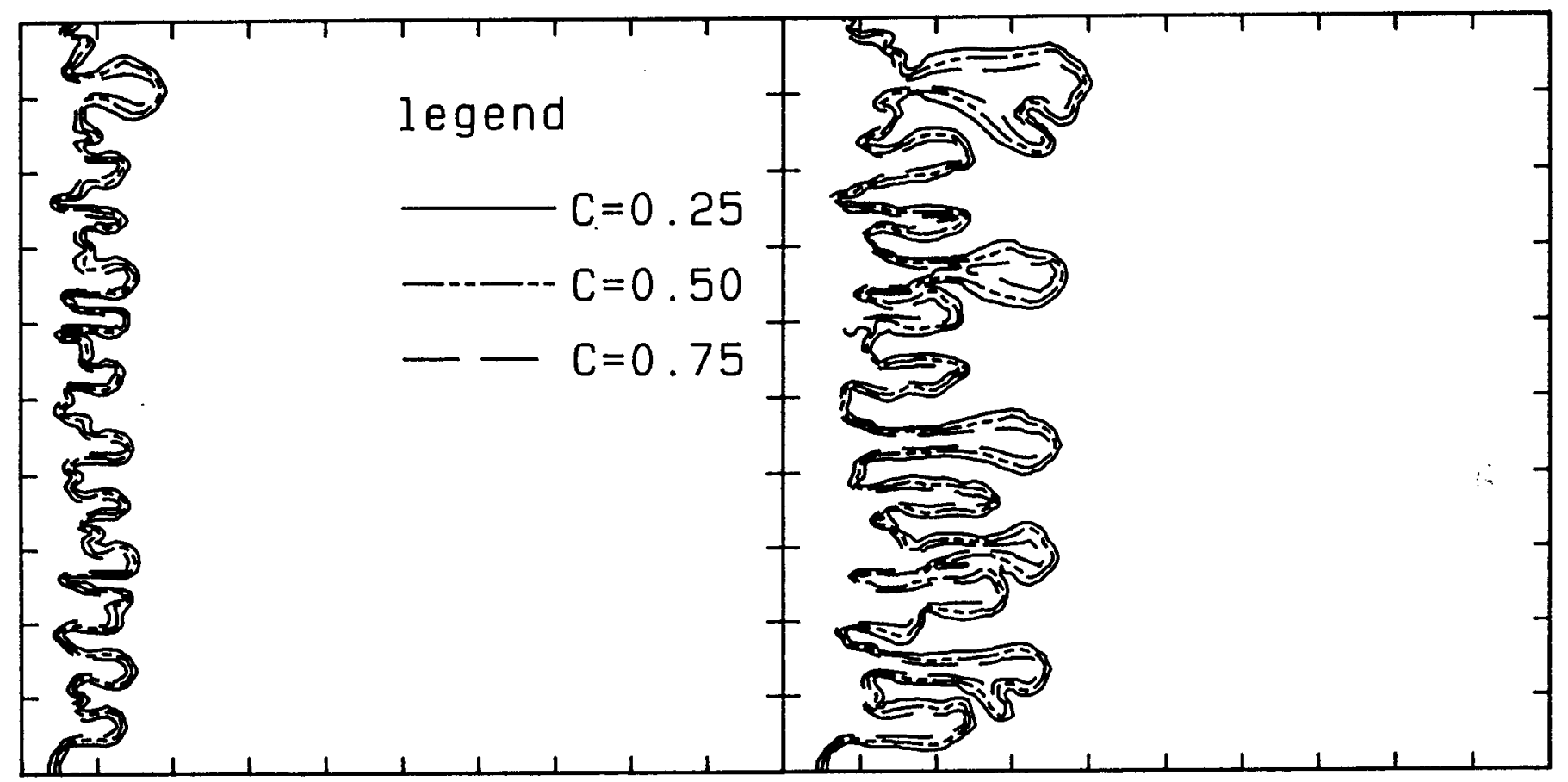

(a) $t=0.1$

(b) $t=0.2$

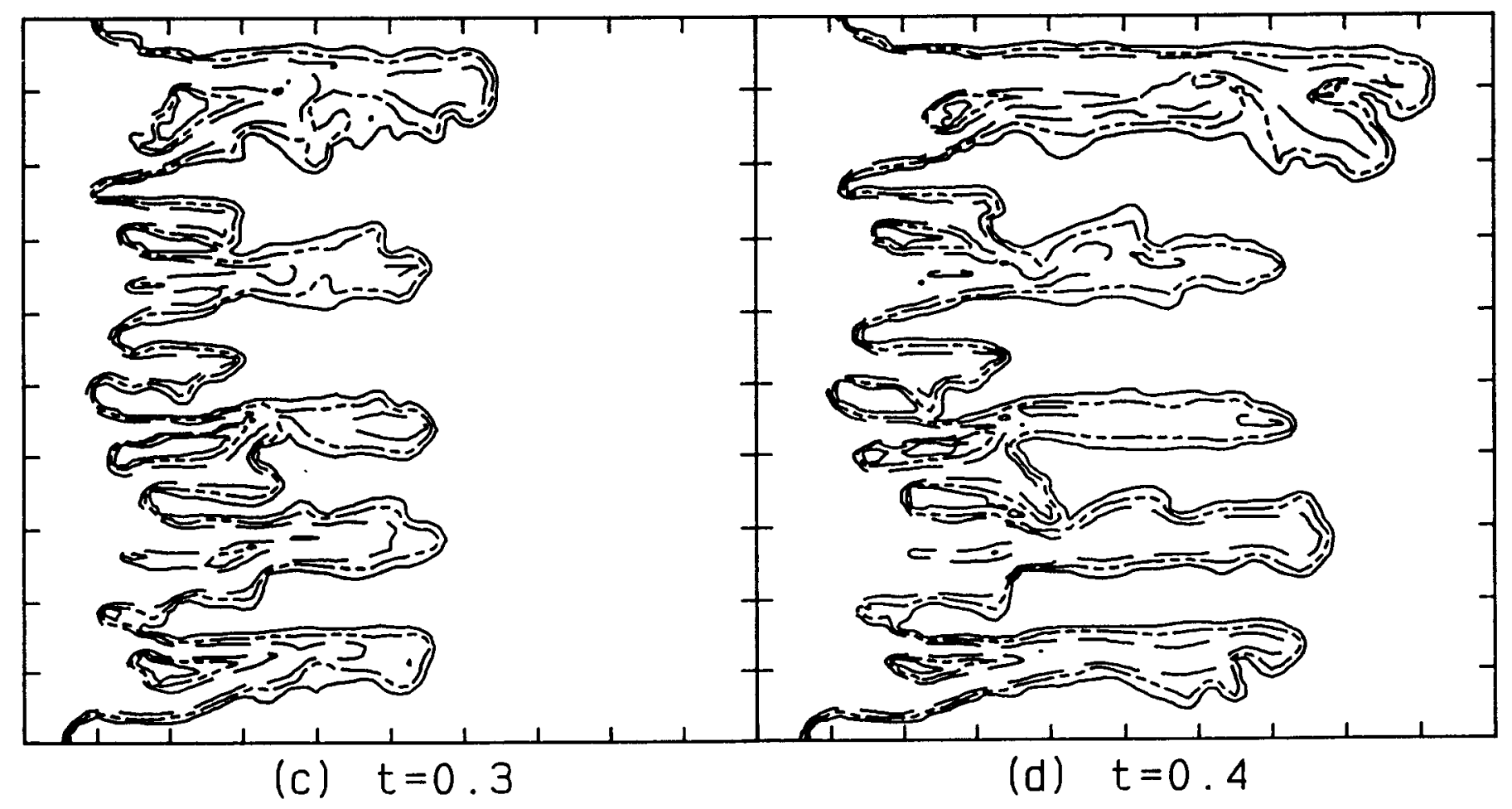

Fig 2. Simulation of base case, $M=75$ (Run 1) 


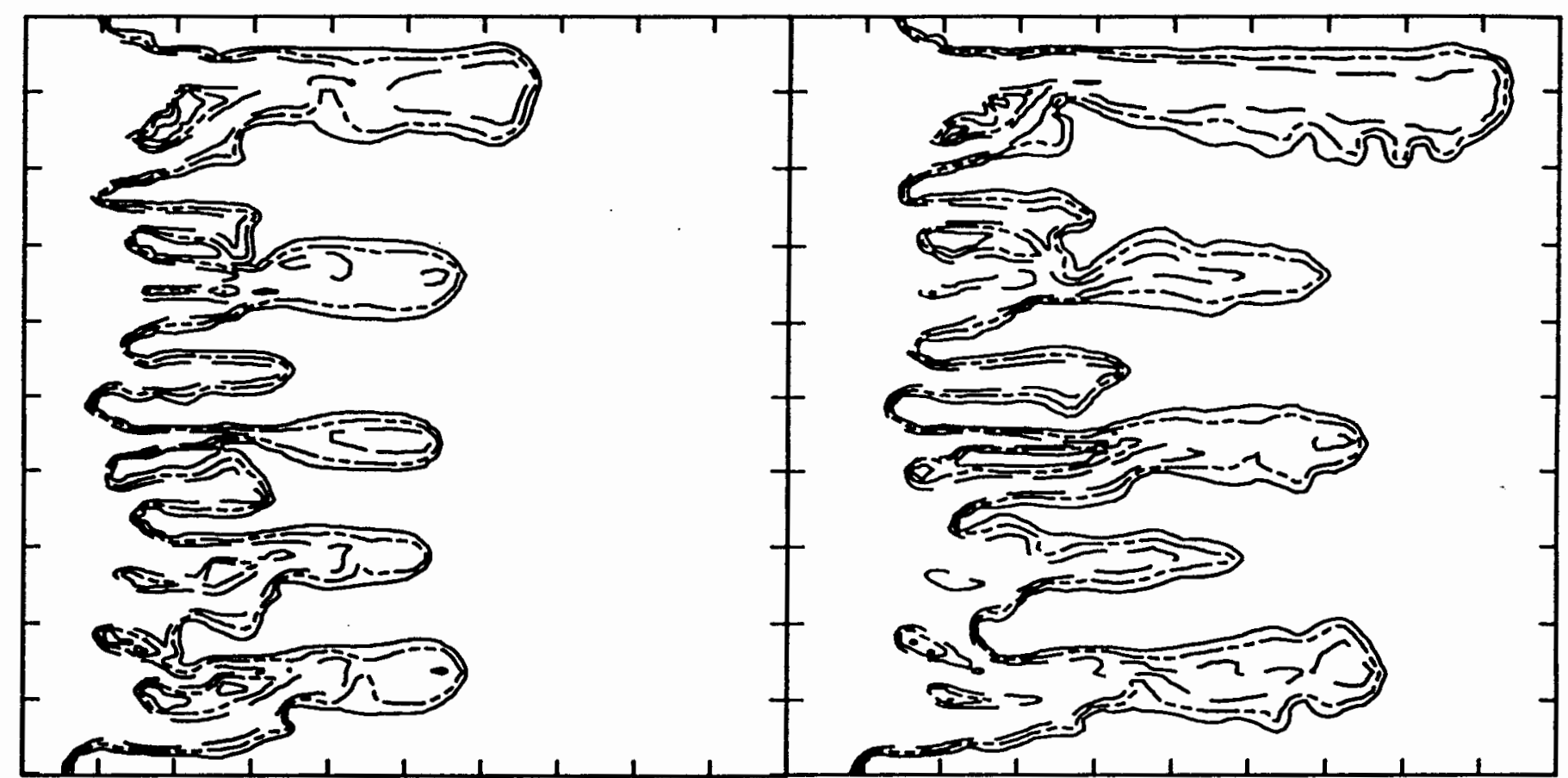

Fig 3. Uniform permeability downstream (Aun 2), $t=0.3$

Fig 4. Heterogeneity reduced by $50 \%$ (Run 4), $t=0.4$



Fig 5. Heterogeneity increased by $50 \%$ (Run 5 ). $t=0.4$

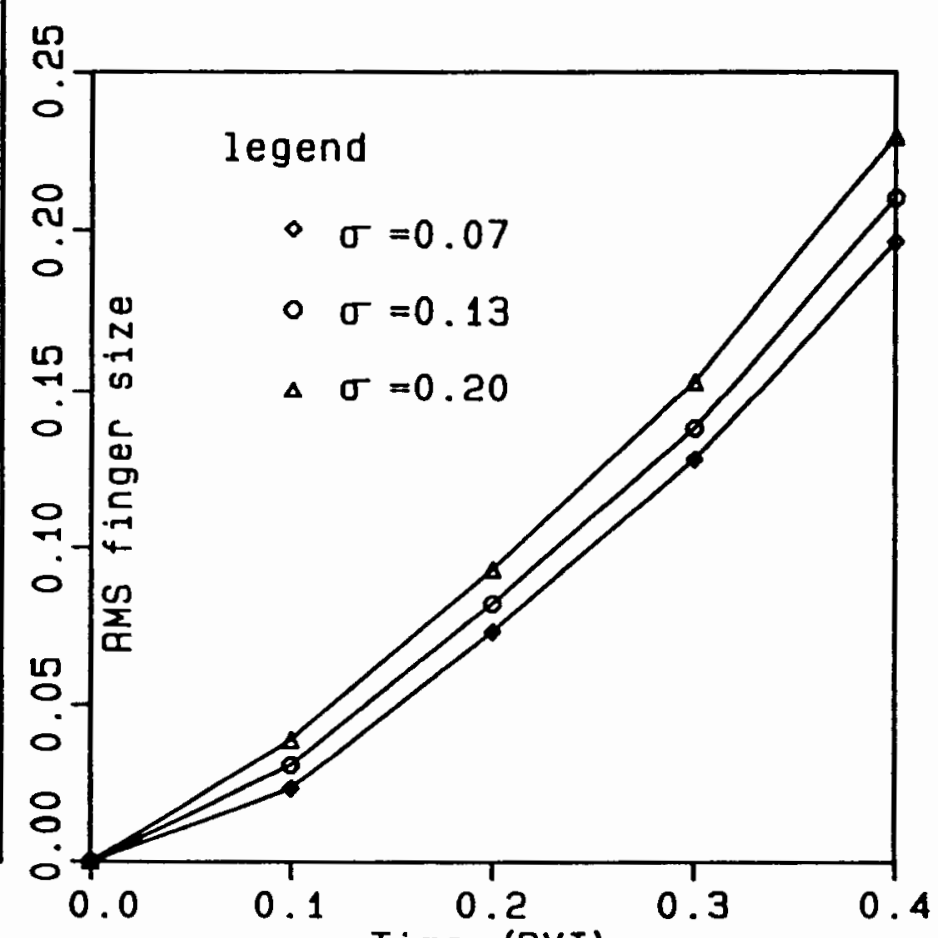

Time (PVI)

Fig 6. Effect of heterogeneity on RMS finger growth rate 


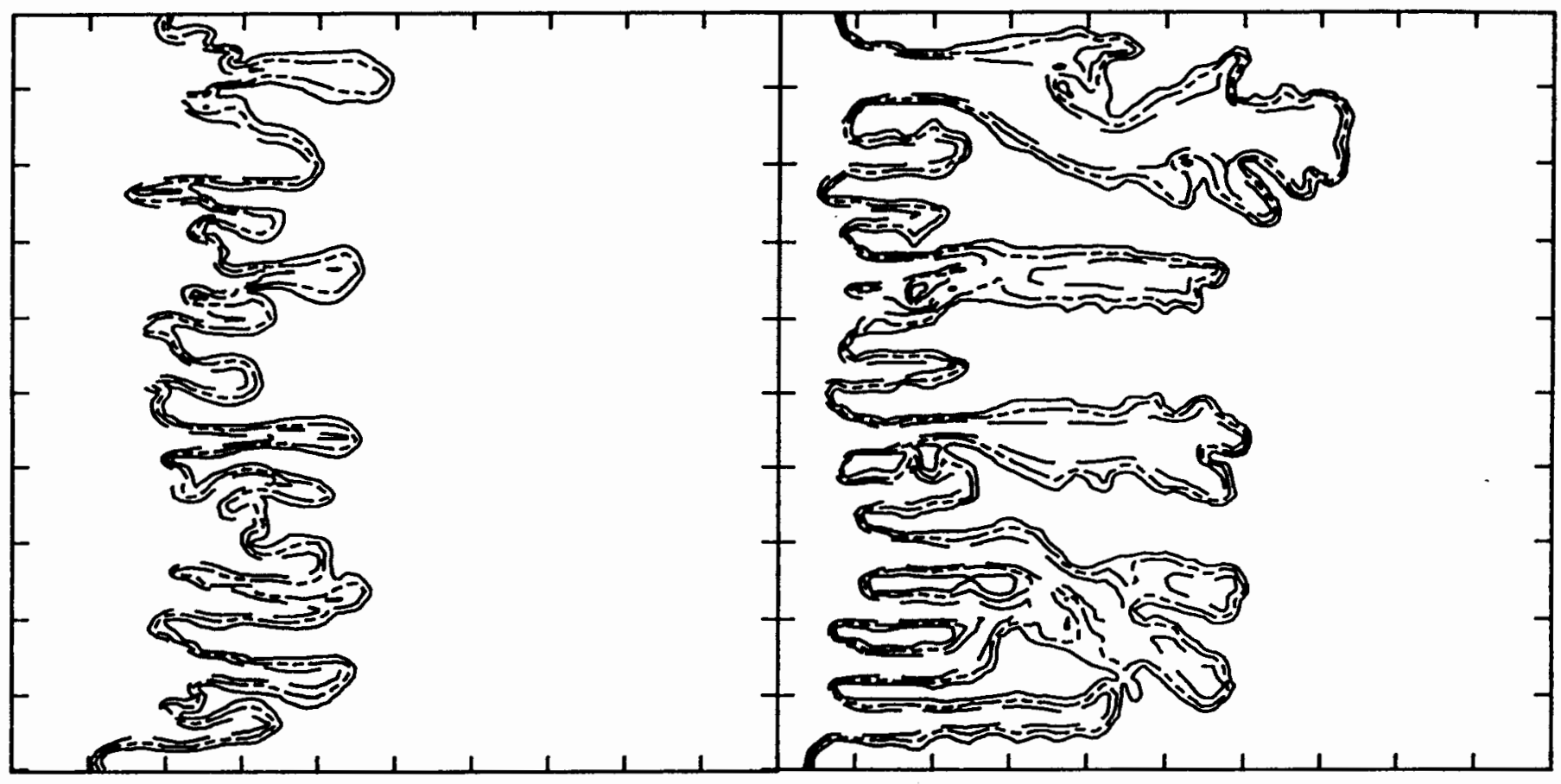

Fig 7. $M=7.5, \quad t=0.3$ (Run 6)

Fig $8 . \quad M=750, t=0.3$ (Run 7)

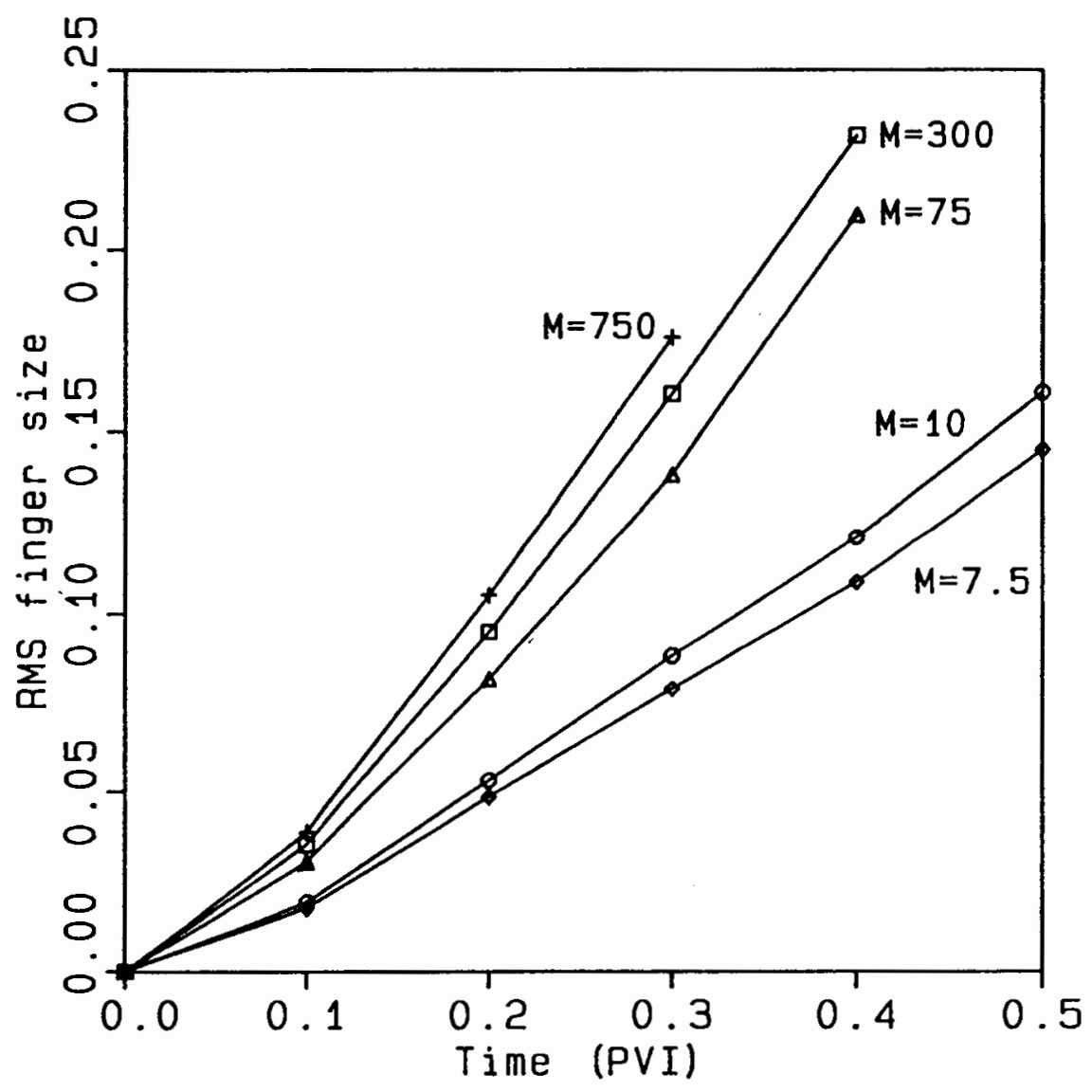

Fig. 9. Effect of mobility ratio on RMS finger growth rate 




Fig 10. Dispersion 5 times higher, $t=0.3$ (Run 8 )

Fig 11. Transverse dispersion 10 times higher, $M=7.5, t=0.3 \quad$ (Run 9)

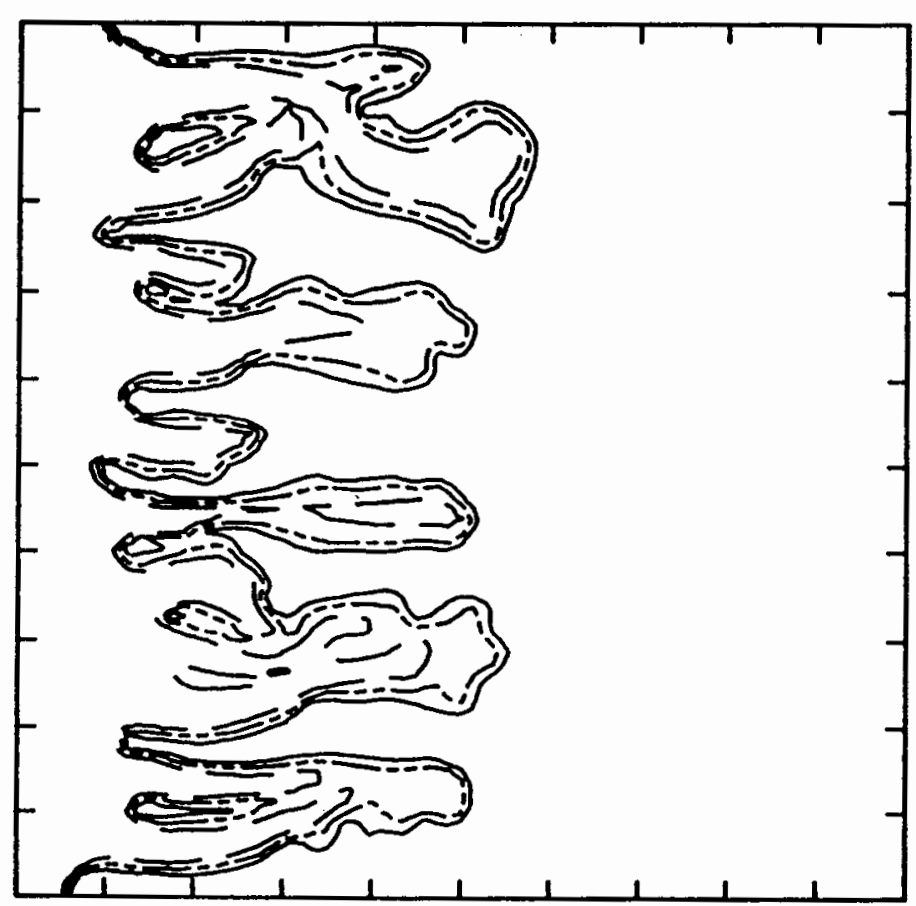

Fig 12. Transverse dispersion 10 times higher. $M=75, t=0.3$ (Run 10)



Fig 13. Effect of transverse dispersion on RMS growth rate 


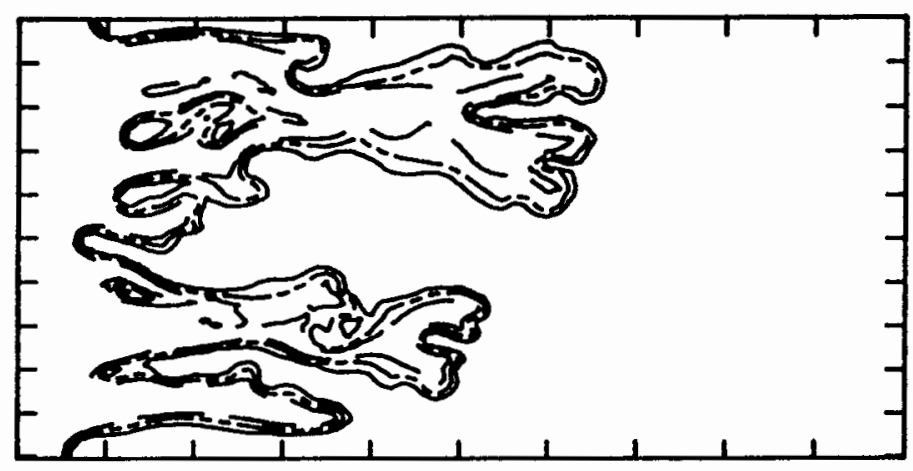

Fig 14. $a=2, \quad M=75, t=0.3$ (Run 11)

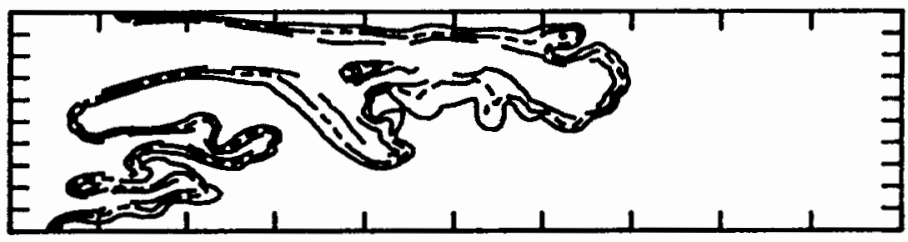

Fig 15. $a=4, \quad M=75, \quad t=0.3$ (Run 12)

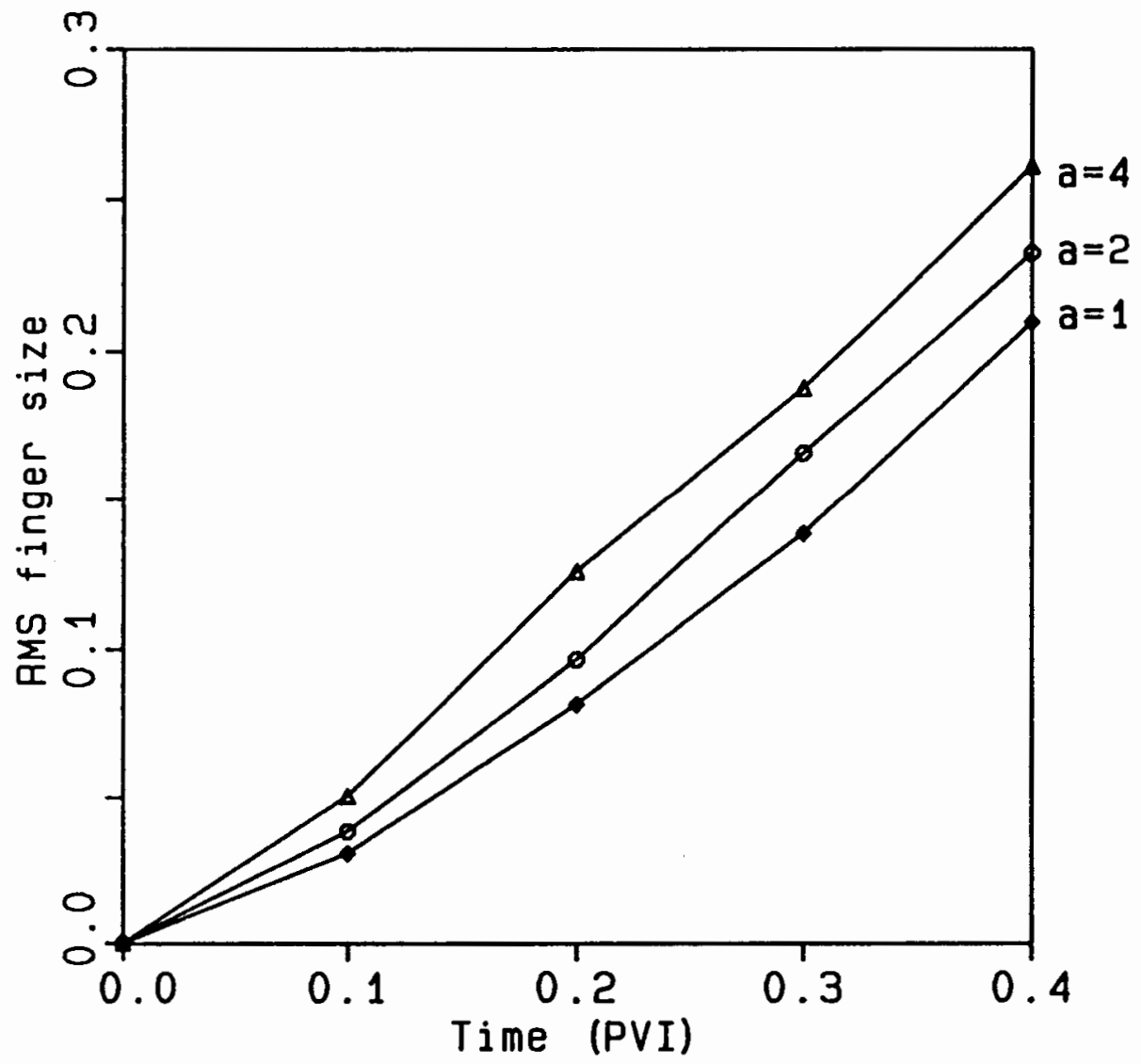

Fig. 16. Effect of aspect ratio on RMS finger growth rate 
